\title{
Phase transition in a static granular system
}

\author{
Matthias Schröter, ${ }^{1, \text { * }}$ Sibylle Nägle ${ }^{1}$ Charles Radin, ${ }^{2, \text { 由 }}$ and Harry L. Swinney ${ }^{1}$ \\ ${ }^{1}$ Center for Nonlinear Dynamics and Department of Physics, \\ The University of Texas at Austin, Austin, Texas 78712, USA \\ ${ }^{2}$ Mathematics Department University of Texas at Austin, Austin TX 78712
}

(Dated: May 2, 2019)

\begin{abstract}
We find that a column of glass beads exhibits a well-defined transition between two phases that differ in their resistance to shear. Pulses of fluidization are used to prepare static states with welldefined particle volume fractions $\phi$ in the range $0.57-0.63$. The resistance to shear is determined by slowly inserting a rod into the column of beads. The transition occurs at $\phi=0.60$ for a range of speeds of the rod.
\end{abstract}

PACS numbers: 45.70.Cc, 81.05.Rm, 45.70.-n

A static assembly of granules, for instance sand in a rigid container, responds differently to shear when packed loosely from when packed tightly [1]. It is natural to enquire whether these two states are smoothly connected as volume fraction varies, or, as with assemblies of particles in thermal equilibrium, are such states sharply separated by one or more phase transitions. We use recent advances in controlling the preparation of granular assemblies to show that the latter holds.

An old magic trick is based on the qualitative difference in the resistance to shear of loosely packed and tightly packed particles: When a pot with a narrow neck is loosely filled with grains, a rod is easily inserted and withdrawn. The rod is then inserted and the grains are shaken or otherwise agitated to a denser state, whereupon the whole apparatus can be lifted by the rod and spun about the performer's head 2, 3].

The existence of distinct phases in granular matter has been widely discussed, but a sharp distinction between the two phases has remained elusive [4, [5, [], the distinction being hampered by the difficulty in preparing a well-defined initial state [6]. The effort to overcome this was advanced significantly by Nowak et al. [7], who used a mechanical tapping protocol to obtain well-defined volume fractions $\phi$ in the range $0.628-0.658$. Recently Schröter et al. [8] showed that a protocol based on expanding the granular medium by pulses of fluid from below could be used to prepare a column of grains with $\phi$ defined to within $0.1 \%$. Using this technique, we prepare granular samples in the range $0.571<\phi<0.633$.

Experiment. - To measure the force of resistance to the insertion of a rod into a granular sample we use an apparatus similar to that in [9, 10, 11]. Those previous studies focused on the influence of geometrical factors such as the size of particles, rod, and vessel, and on how the penetration force increased when the rod approached the bottom boundary. Those experiments were performed at a single volume fraction, $\phi=0.59$ [9, 10].

Our measurements are performed with a home built granular penetrometer: a translation stage (driven by a stepper motor with a step size $2.5 \mu \mathrm{m}$ ) moves a stainless steel rod (diameter $6.3 \mathrm{~mm}$ and flat head) downwards into a granular sample. The force needed for penetration is measured with a load cell with a full range of $10 \mathrm{~N}$ (Honeywell, Model 31). The sample consists of soda lime glass beads from Cataphote with a diameter of $265 \pm$ $15 \mu \mathrm{m}$ and a density of $2.484 \pm 0.002 \mathrm{~g} / \mathrm{cm}^{3}$ (measured with a Micromeritics gas pycnometer AccuPhys 1330). The beads are contained in a water-fluidized bed where flow pulses of different flow rates allow us to select a volume fraction $\phi$ for the static sedimented bed [8]. (If air rather than water is used to fluidize a bed, it is difficult to obtain low enough volume fraction to see the transition 12.).) The beads are fluidized inside a square bore glass tube $\left(39.9 \times 39.9 \mathrm{~mm}^{2}\right)$. The ratio of inner tube size to rod diameter is 6.3 , larger than the value five that $[9]$ found to be sufficient so that the influence of the vessel walls was negligible. Flow pulses are generated using a digital gear pump (Barnant Co., model no. 75211). The volume fraction is determined from measurement of the bed height; one pixel in the digital images corresponds to a change of only $0.02 \%$ in $\phi$.

Results. - The force on the penetrating rod increases monotonically with its depth, as Fig. 1 (a) illustrates. Contrary to the observations in [11], we find that the rate of growth is not polynomial (see the double logarithmic plot in Fig. 1 (b)). Further, at maximum depth the rod is $40 \mathrm{~mm}$ from the bottom of the container, well beyond the distance of $20 \mathrm{~mm}$ where boundary effects have been found to be measurable for conditions comparable to our experiment [9].

The rate of change of force with volume fraction exhibits a well-defined transition, which occurs for $\phi=$ 0.598 for a rod depth of $60 \mathrm{~mm}$, as Fig. [2]illustrates. The $\phi$ values corresponding to the transition increase slowly with rod depth (cf. inset of Fig. 22). This dependence of the transition point on the penetration depth cannot correspond to a dependence of $\phi$ on depth because then $\phi$ would have to decrease with depth, which is unphysical.

There is no hysteresis in the transition: measurements at different volume fractions can be made in any order, without affecting the results. However, the value of $\phi$ at 

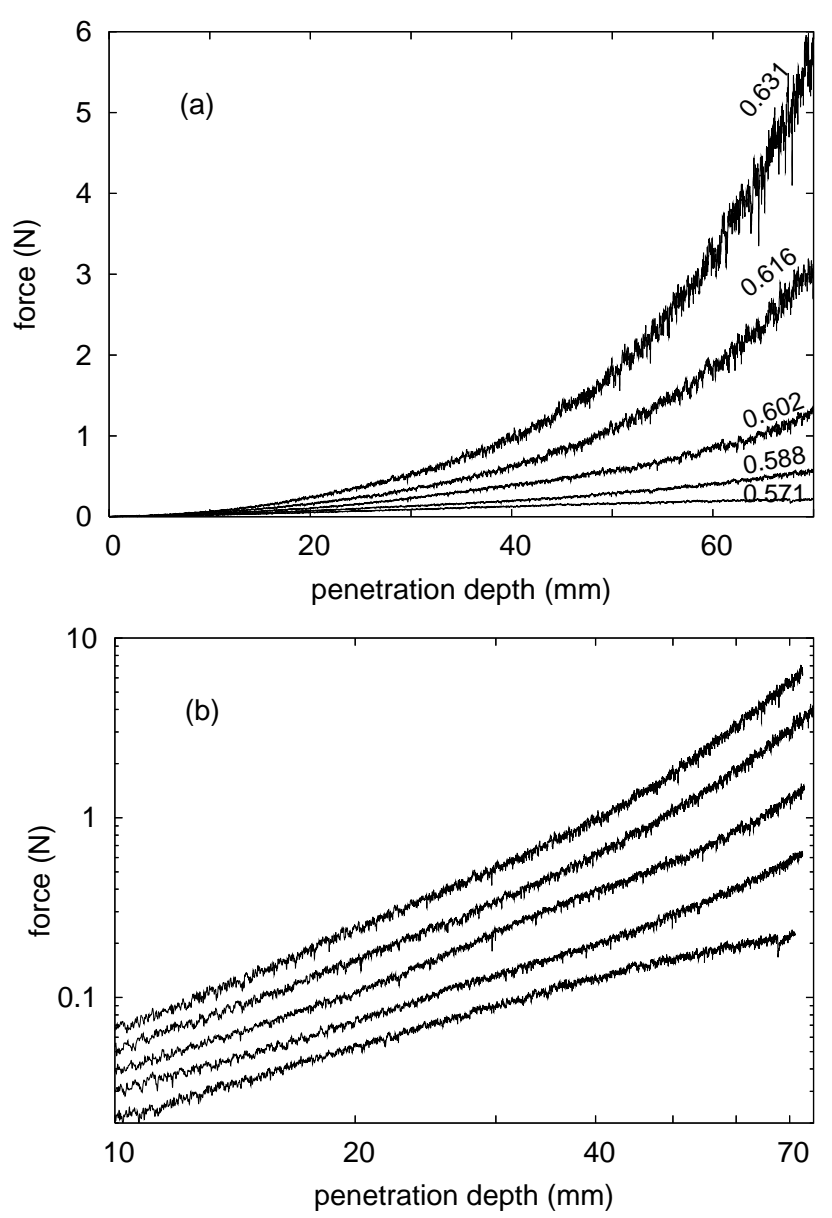

FIG. 1: (a) Penetration force as a function of depth for different volume fractions. (b) Double logarithmic plot of the data in (a). These curves are measured with a penetration speed of $10 \mathrm{~mm} / \mathrm{s}$, but the results are the same for a range of penetration speeds. The total sample height is $110 \mathrm{~mm}$ (at $\phi$ $=0.6)$.

the transition depends weakly on the frictional characteristics of the grains, which can change slowly with usage of the beads [8].

The penetration force is independent of the speed of the rod (cf. Fig. 2). The absence of a dependence on penetration speed is in agreement with the results of [9] at $\phi=0.59$. It also shows that the flow of water induced by the penetrating rod does not alter the results, in agreement with [1].

Typically, only about five percent of the force we measure is exerted on the sides of the rod, as we see from Figure 3 which measures the force on withdrawal. The measurements during withdrawal also suggest the phase transition, though it is not well defined since the forces are so much smaller.

The transition between distinct phases indicated by Fig. 2 should be accompanied by changes in other prop-

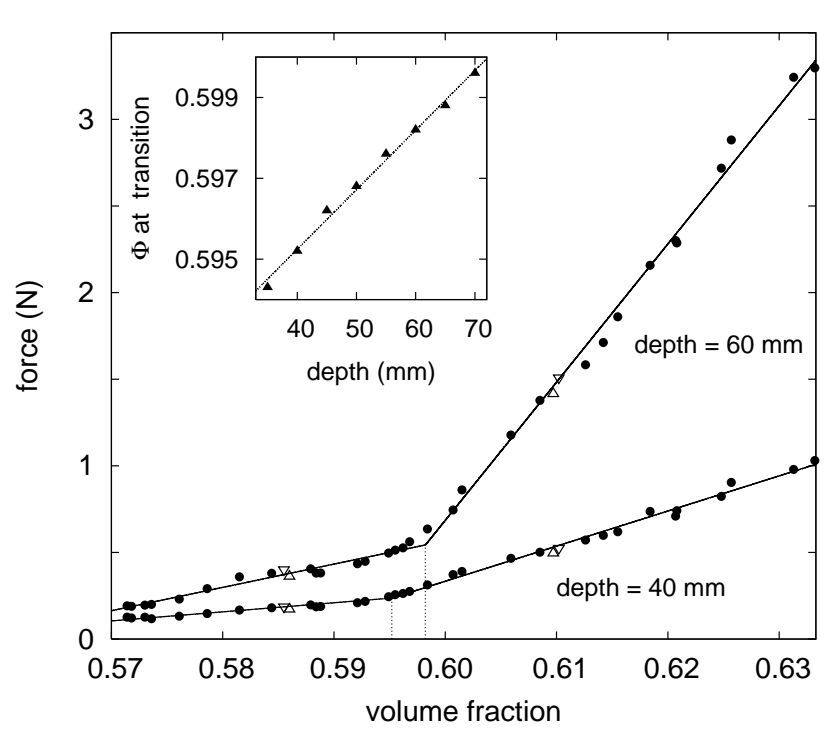

FIG. 2: The dependence of the penetration force on volume fraction changes at $\phi=0.598$ at a depth of $60 \mathrm{~mm}$. Data represented by $\bullet$ were measured at a penetration speed of 10 $\mathrm{mm} / \mathrm{s}, \nabla$ at $5 \mathrm{~mm} / \mathrm{min}$ and $\triangle$ at $20 \mathrm{~mm} / \mathrm{min}$. The transition points are determined by the intersection of least-square fits for the volume fractions below 0.595 and above 0.6 . The inset displays the dependence of the transition point on the penetration depth.

erties. Indeed, measurements of the average height of the bed as a function of $\phi$ reveal, as Fig. [4 shows, a transition at the same $\phi$ as in the force measurements.

Discussion.- A system similar to ours whose phase transitions have been studied is a collection of colloidal particles prepared at different $\phi$ by centrifugation [13]. The colloidal system has been characterized by a freezing density $\phi_{f}=0.407$ and a melting density $\phi_{m}=0.442$, such that for $\phi<\phi_{f}$ the system is a fluid, for $\phi>\phi_{m}$ the system is a crystalline solid, and for $\phi_{f}<\phi<\phi_{m}$ the system is a mixture of the two states. This phase behavior (but with $\phi_{f}=0.494$ and $\phi_{m}=0.545$ ) was found earlier in Monte Carlo and molecular dynamics simulations for frictionless hard spheres [14].

There are differences between a colloidal system and our static granular system. For example, the colloidal system has pure solid and fluid phases separated by a coexistence region with a sharp transition at each end, while the granular system exhibits only one transition in our experiment. Another difference is that the transition in the colloidal system corresponds to a change of symmetry from disordered to crystalline, while the transition in the granular system is marked by a change in the resistance to shear. However, a recent consideration of the transition in the equilibrium hard sphere model [15] suggests that the symmetry change may be hiding the fundamental mechanism in that model, a type of ge- 


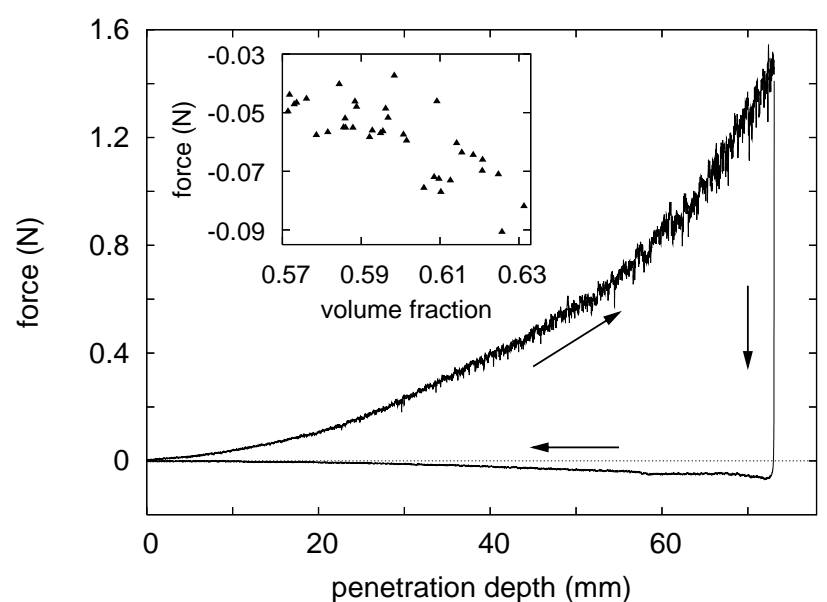

FIG. 3: Forces measured during a full cycle of insertion and withdrawal at a volume fraction $\phi=0.602$. The speed of the rod is $10 \mathrm{~mm} / \mathrm{min}$ in both directions. The inset shows the withrawal force measured at a depth of $70 \mathrm{~mm}$ for the same experiments as in Fig. 2

ometric constraint, which may also underlie the phases of the granular system.

The volume fraction at which we observe a transition coincides with earlier observations of interesting behavior. In [8] volume fluctuations around a static steady state were measured using a series of identical flow pulses in a fluidized bed, and those fluctuations were found to exhibit a parabolic minimum at volume fractions between 0.587 and 0.596 , depending on the surface roughness of the beads. Arguments based on the central limit theorem showed that this minimum corresponds to a minimal number of beads being contained in a statistically independent region, which is tantamount to a minimum in the correlation length. Another study found that if a fluidized bed is slowly defluidized, it exhibits a behavior similar to that of a supercooled liquid, with an arrest transition at $\phi=0.594$ 16. At the arrest transition $\phi$ became nearly independent of the flow rate and the correlation length went to zero. A similar result has been obtained in an analysis of the Delauney tesselation of tomograms of sphere packings: at volume fractions between 0.58 and 0.60 re-adjustments involving only a single sphere become impossible and any dynamics requires collective and correlated motion of larger sets of spheres [17]. And finally, as indicated by Figure 4 our transition takes place at the critical state density of soil mechanics 1]; at densities below this the granular sample collapses under stress, while at densities above this it expands under stress.

In conclusion, we have taken advantage of a method to produce beds of granules with well-defined volume fractions to search for a phase transition as a function of

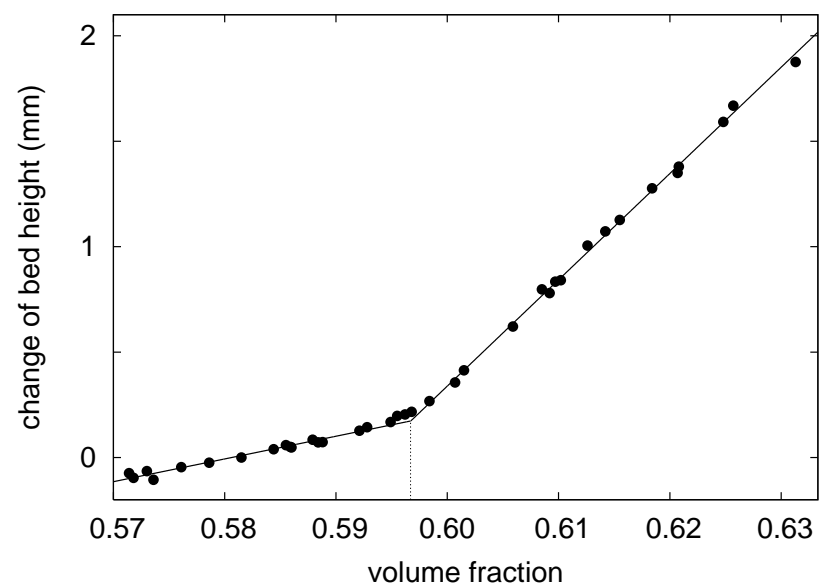

FIG. 4: Change in the height of the bed relative to the original bed height for a rod inserted to a depth of $60 \mathrm{~mm}$. The transition occurs at $\phi=0.597$. The bed is slightly deformed near the rod but not sufficiently to change the average height of the bed.

volume fraction. The transition revealed by our measurements of penetration force and bed height should help in the understanding of granular behavior, for instance, in avalanches 18. The existence of a well-defined transition between two phases also suggests the appropriateness of a statistical approach to understanding the phases of granular matter.

We thank Persi Diaconis, Pierre Evesque, Daniel Goldman, W.D. McCormick, and Peter Schiffer for helpful discussions. Schröter and Swinney were supported in part by the Robert A. Welch Foundation; Radin was supported in part by NFS grant DMS-0352999.

* Electronic address: schroeter@chaos.utexas.edu

† Electronic address: radin@math.utexas.edu

[1] R. M. Nedderman, Statics and Kinematics of Granular Materials (Cambridge University Press, 1992).

[2] L. H. Branson, Indian Conjuring (E.P. Dutton, New York, 1922), pp. 39-41.

[3] T. Aste and D. Weaire, The Pursuit of Perfect Packing (Institute of Physics Publishing, 2000).

[4] H. M. Jaeger, S. R. Nagel, and R. P. Behringer, Rev. Mod. Phys. 68, 1259 (1996).

[5] L. P. Kadanoff, Rev. Mod. Phys. 71, 435 (1999).

[6] P. G. de Gennes, Rev. Mod. Phys. 71, S374 (1999).

[7] E. R. Nowak, J. B. Knight, E. Ben-Naim, H. M. Jaeger, and S. R. Nagel, Phys. Rev. E 57, 1971 (1998).

[8] M. Schröter, D. I. Goldman, and H. L. Swinney, Phys. Rev. E 71, 030301(R) (2005).

[9] M. B. Stone, R. Barry, D. P. Bernstein, M. D. Pelc, Y. K. Tsui, and P. Schiffer, Phys. Rev. E 70, 041301 (2004).

[10] M. B. Stone, D. P. Bernstein, R. Barry, M. D. Pelc, Y. K. 
Tsui, and P. Schiffer, Nature 427, 503 (2004).

[11] G. Hill, S. Yeung, and S. A. Koehler, Europhys. Lett. 72, 137 (2005).

[12] R. Ojha, N. Menon, and D. J. Durian, Phys. Rev. E 62, $4442(2000)$.

[13] P. N. Pusey and W. V. Megen, Nature 320, 340 (1986).

[14] D. Frenkel and B. Smit, Understanding Molecular Simulation (Academic Press, San Diego, 2002), 2nd ed.

[15] L. Bowen, R. Lyons, C. Radin, and P. Winkler, Phys.
Rev. Lett. 96, 025701 (2006).

[16] D. I. Goldman and H. L. Swinney, Phys. Rev. Lett. 96, 145702 (2006).

[17] T. Aste, J. Phys.: Condens. Matter 17, S2361 (2005).

[18] P. Evesque, Phys. Rev. A 43, 2720 (1991)); P. Evesque, D. Fargeix, P. Habib, M. P. Luong, and P. Porion, Phys. Rev. E 47, 2326 (1993). 